\title{
Use of Surgical Drains in Gynaecology Surgery Revisited
}

Ho Ping Ling, cC Khong

1 Department of Obstetrics and Gynaecology, KK women's and Children's Hospital, Singapore

\section{Introduction}

Post-operative haemorrhage remains a significant complication of surgery worldwide, associated with high mortality and morbidity. Haemorrhage within the first 48 hours, also called reactionary haemorrhage, is usually due to displacement of clot in a vessel, or slipped ligature. These may happen secondary to mobilization, increase in blood pressure postoperatively, resulting in re-opening of vessels or slipping of ligatures, resulting in varying degrees of internal haemorrhage. The role of surgical drain in these cases are of utmost important in early identification of internal bleeding. Our experience suggests surgical drain use is a novel technique used in various surgical procedures, with best intention, for prophylaxis or for treatment. Current evidence is controversial to support routine prophylactic use of surgical drain after gynaecological surgical procedures.

\section{Discussion}

Post operative internal haemorrhage is a potentially lifethreatening event. Surgical drain insertion is a relatively simple and effective method which can be routinely adapted in the monitoring of post-operative haemorrhage. In most cases, prophylactic surgical drain insertion enables early identification of internal haemorrhage, decompression of wound bed, thus reducing morbidity and mortality of patients in addition to enhancing wound recovery. In both our cases, haemostasis was thoroughly secured and optimal prior closure. We postulate that the underlying pathology may be dislodgement of clots or reopening of vessels which were ligated or diathermised. Surgical drain insertion in these cases enabled identification of internal haemorrhage prior decompensation. Although, prophylactic surgical drain is not a substitute for meticulous surgical technique.

Reference:

Hurrell A. et al. Evaluation of the selective use of abdomino-pelvic drains at laparoscopic myomectomy in enhanced recovery, do drains delay discharge home? EJOG 2015 Feb; 185:36-40.

$\mathrm{Kim} \mathrm{Si}$ et al. Benefit of negative pressure drain within surgical wound after cytoreductive surgery for ovarian cancer. Int. J. Gynecol. Cancer. 2015 Jan;25(1):145-51.

Kim Si. et al. Application of a subcutaneous negative pressure drain without subcutaneous suture: impact on wound healing in gynecologic surgery. EJOG 2014 Feb;173:94-100

\section{Case Scenario 1}

A 43 year-old woman underwent elective total abdominal hysterectomy for large uterine fibroids. She has no known other surgical or medical history. Surgery was uncomplicated, haemostasis was optimal.A Jackson-prett surgical drain was inserted. She remained haemodynamically stable post operatively, with no obvious signs of acute internal haemorrhage. On post op day-2, there were sudden increase of drain output consisting of frank blood. Her haemoglobin dropped to $6 \mathrm{~g} / \mathrm{dL}$. During relaparotomy, a small ooze detected near right infundibulopelvic ligament, which was arrested by diathermt. Patient recovered well.

\section{Case Scenario 2}

A 52 year-old lady underwent open myomectomy for large solitary fibroid. Surgery was uncomplicated, haemostasis secured prior closure. At 8 hours post-operatively, surgical drain output of 500mililitres frank blood detected through a Jackson-pratt drain. Patient remained haemodynamically stable. No omnious clinical signs and symptoms of acute internal haemorrhage. During re-laparotomy a small arterial bleeding was identified and arrested at the bladder base. Patient recovered well.

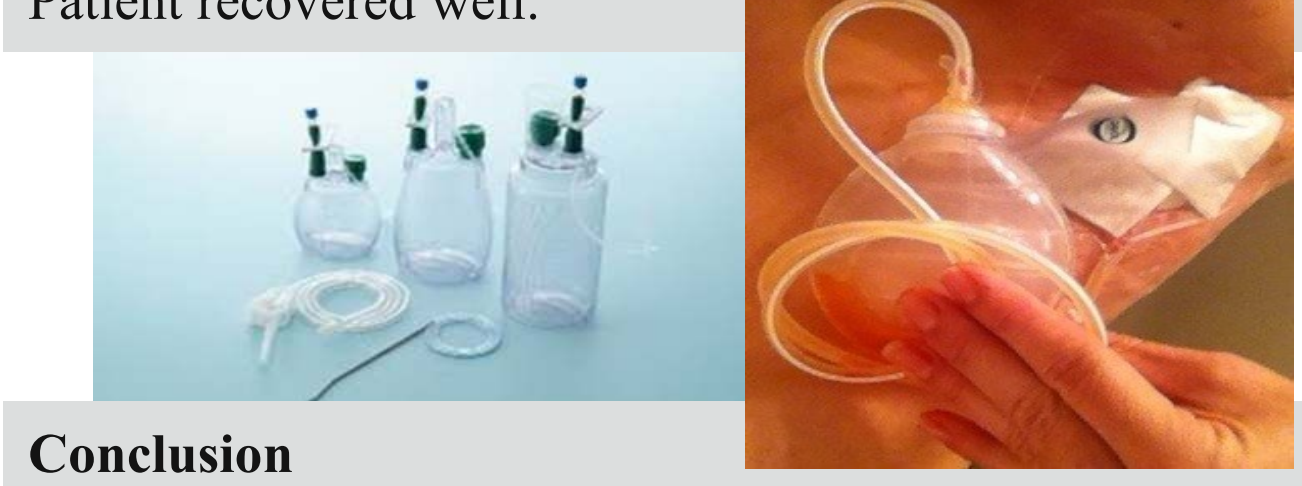

It is said, "Nobody has regretted putting in a drain but many have regretted not putting one in after surgery". The art of surgery aims for minimal blood loss during operation and zero loss after that. This involves a degree of faith in both surgical skills and patients' haemostatic mechanisms. By the time haemodynamic deterioration occurs, significant morbidity might have set in. A surgical drain pose hardly any increase in surgical time or infection that delays patients' discharge. We conclude that routine use of surgical drain can reduce morbidity from unanticipated postoperative haemorrhage.

PATIENTS. AT THE HE RT OF ALL WE DO. 